\title{
Study on the Role of Scene Perspective in Animation Production
}

\author{
Bingxu Ni ${ }^{1, *}$ \\ ${ }^{1}$ Fuzhou University of International Studies and Trade, Fuzhou, Fujian, China \\ "Corresponding author. Email: 2662046667@qq.com

\begin{abstract}
Perspective plays a very important role in animation production. Its combination with photography lens can not only improve the richness of the picture, but also reflect more technical content and aesthetic feeling. The use of perspective in two-dimensional space can enhance the depth and visual strength of the space, thus making the characters in the animation scene more vivid. Through the analysis of perspective relation, this paper helps scene perspective more and better applied in the animation production.
\end{abstract}

Keywords: scene perspective, lens and atmosphere, physical sense of space and psychological sense of space

\section{INTRODUCTION}

An excellent animation design work should not only have a readable script, lens and action design of the characters, but also provide a stage for the role performance corresponding to them. This stage is the space for the interpretation of the animation characters, that is, the scene of the animation. Scene design in the production of animation can not only contribute to a good visual effect in the animation, but also to foil the plot development and character personality. From the technical level, the animation visual effect often comes from the reasonable use of the field-scene perspective. A good scene concept designer's grasp of the scene will also be reflected in this perspective.

\section{THE RELATIONSHIP BETWEEN SCENE PERSPECTIVE AND ANIMATION}

\section{A. The importance of scene design in animation}

Scene design is a very rigorous and demanding part in animation works. It is a stage and background designed for the story development process of animation according to the existing script, storyboard, role characteristics and background. In the design, the scene painter will use a variety of expression techniques to depict the picture. For example, the natural environment, indoor and outdoor landscape will be elaborated and sketched according to plot changes and character needs, and of course, it also true of the color and tone. The rational use of the perspective principle is very important. It can not only objectively present the picture imagined by the painter and produce a richer animation scene effect, but also shorten the sense of immediacy of the viewing object, fill its abstract psychological space and stimulate the compression and expansion of viewers' emotions. It can be said that the use of scene perspective is very critical and not to be underestimated.

Here is a myth that must be corrected. Some people think that the scene refers to the background, which is superficial. In theory, the animation scene is a concept of the mutual combination and setting of time and space, as well as a three-dimensional performance space on which the characters limited by the script live, and is closely related to the story and the characters. Therefore, scene design is an important part of animation works, not dispensable, nor does it serve as a simple background. Its quality determines the quality of the work, and its success or failure is directly related to the success or failure of animation products.

\section{B. The relationship between perspective and lens in an animated scene}

Scene perspective method, which is often used in animation production, can be divided into two types: geometric perspective and air perspective.

The so-called geometric perspective is the shape, size and distance of the object; air perspective refers to the visual induction and changes that take place between the color of an object reflected in the retina through the air. The animation images and films we see are all conveyed through the lens. The ideas and contents the director wants to present to the audience are realized through the images after the processing of relevant technologies. Simply speaking, such images are shots.

There is a major difference between scene and lens. Relatively speaking, the lens is just a single picture that 
I call "amplitude" or "frame", which has the concept of "quantity". The scene corresponds to a certain relationship between time and space, which has the meaning of "quality" Whether an animated image or a film is composed of countless lens systems to complete the story to be told. It can be said that in the story narration, the lens is the most basic fixed format, and the scene is the basic elements of the story, which can be regarded as a major part of the story core.

In addition to providing the linear proportion of distance and size to the lens, the designer also needs to incorporate the use of asymmetric distorted perspective when designing the scene. This concept is relatively complex, and is usually the experience of dynamic color and transformation art. It is closely related to the plot narration and the fate of the characters. The production of such a scene will enhance the hidden visual strength of the work and leave a deep impression on the audience. Of course, in order to achieve the shooting effect, it is also necessary to choose different lens equipment, such as wide-angle lens, which makes the processing of the scene closer to the requirements of visual effects. The use of wide-angle lens will bring unusual visual experience. The focal length is smaller than the ordinary lens, but the perspective is wider and the depth of field varies greatly. The works shot can give people a certain impact and play a very good supporting role in the overall planning and processing of the scene. Therefore, it is not difficult to see that it is also very important to learn and master the relevant photography knowledge, which is a combination of technology and art, in order to find a balance between the quality and quantity of animation scenes, and ultimately achieve a more magical effect.

In addition to the full-shot, it is also common to use the scenery shot in the animation production. According to the different shooting objects, it can be divided into the view-empty shots and the object-empty shots. The view-empty shots are mainly used to in animation to explain some information. The time and place of the story can be explained clearly through the transformation of the lens, so that the audience can be clearer about the change of time. Generally, the scene is especially prominent in such a shot, which adopts the long shot or the extreme long shot. However, the object-empty shot is used to connect the transformation of characters and events. As a means of transition between shots, as well as a common method in daily use, it is mostly performed by close shot or big close-up in general. The scene here is basically materialized, and notably it does not refer to the background. No matter what kind of view-empty shot is used, it can constantly enrich the visual effect of animation works and enhance the sense of rhythm and scene interest. The role played by scenery shots is foil and reinforce, which is not distracting.
Meanwhile, the role of long shot cannot be underestimated. The use of the long shot is a shot with consistent scene all along. And if not edited, its length will not be limited. It is not a simple thing to present a complete scene in an animated image and at the same time to ensure its fluency. A coherent shot like this requires a lot of skill in production. Because its perspective technique is tested at every frame, therefore drawing such a coherent scene is a time-consuming task.

With the continuous development of science and technology, 3D technology has also developed rapidly. The production of long shots through 3D technology can effectively save the production cost of animated films.

To sum up, attention must be paid not to use the long shot too much in the scene design. The push and pull without change will make viewers feel visual fatigue and confused about the main body of the shot. Therefore, the use of the long shot is very strict, and it is only used in special cases to complete the scene shooting.

\section{THE RELATIONSHIP BETWEEN PERSPECTIVE AND ANIMATION SCENE ATMOSPHERE}

\section{A. Perspective sets off the characters in the animation by contrast}

With the development of the story, the character's space environment is often the node of the whole plot. In order to clearly show the image of the characters in the animation in people's view, it is necessary to darken the elements in the background of the picture and make them relatively blurred in the perspective, so as to highlight the key points and achieve the effect of setting off. The animation scene atmosphere displayed after the processing of the corresponding technology not only brings a sense of excitement, but also can clearly show the characters in the scene, so that people can see the animation effect with more impact. This atmosphere full of air perspective effect plays a very important role of setting off, making the tall even taller and the small even smaller.

In the process of making some animated images, fisheye lens will be used to explain the story. Fisheye lens is the perspective of ultra-wide angle. This distorted perspective technology has high technical requirements for scene painters, and the scene pictures produced will provide the audience more highlights. For example, in the animation " $\mathrm{K}$ ", there is a scene where Misaki is chasing Yashiro. Here, the ultra-wide angle perspective is used to broaden the audience's vision. The camera tracking creates a sense of being in the scene for the audience. 
The use of fisheye lens has the function of visual extension and expansion, allowing the characters to present a visual effect in the space. Although it is an extreme way of expression, it is very attractive to the audience.

\section{B. Perspective can create a sense of space in the scene}

The sense of space, whether in painting or animation, is a real feeling pursued by the creator. For the leading role of the animated image works, it is necessary to have a certain space to carry out their own activities and performances. Long-range perspective and close shot, or sometimes distant and sometimes close shots are all techniques of representing space and have great attraction to the audience. Lack of space in the design will lead to a sense of disorder and no sense of hierarchy.

For scene painters, being able to apply perspective technology well is a reflection of their own skills and qualities. Before the animation painting of the scene, often scene painter has formed a conceptual renderings in the mind, that is, people often say the "mental notes". In the initial drawing of the manuscript, lines are generally used to express the perspective relationship. Through the changes of lines, the perspective effect is displayed in the central position of the vision. Through the simple and vague delineation of the scene space, a more stable and peaceful emotional tone of the animation is created.

There is a technical level of things to explain here: when the scene is designed and painted, the viewfinder should be selected a little larger, in order to prevent the appearance of the scene "goof", and to facilitate a series of modifications in the later stage.

The part of the animation image lens that can be transferred is the internal composition of the entire animation scene. Some large and long background patterns are often used to effectively connect the lens, thus giving the lens a rhythmic narrative. After plane drawing is made, the use of stereoscopic lens can also well shoot a sense of space. For example, in the animated film "Your Name", there is an unforgettable long shot at the beginning, which is to follow the sliding track of the comet and to look up at the sky in the whole scene, creating a vast space distance.

Outstanding designers often form a unique style and have obvious personal characteristics in the way of scene processing. For example, the frequent appearance of the sky, clouds and trees shows warm colors through the rational use of light effect. In terms of scene art style, it can be seen that the scene is influenced by Kazuo Oga. The multi-level scenes are designed and produced to accommodate a variety of camera movements and to make the exterior scenes as large, open and deep as possible. The depth of field is intensified to constantly increase the space tension. In this kind of scene, the reasonable use of shade, shadow and tonal and other auxiliary means is very necessary. In this way, the treatment effect that the close-up is darker, the perspective is brighter, the close-up is more solid and the perspective is more empty can be realized, which not only improves the aesthetic feeling of the whole painting, but also enhances the reflection of the perspective requirements.

The sense of space is generally used intensively in the animation images, mostly in the realistic style, and often seen in other styles of animation. However, the style is different, and the way of selection is also different, mainly depending on the author's basic concept of scene conception. In some flat and abstract animations, in order to show the sense of space, the changes of color, temperature and light and shade, and the color and tone are selectively used.

To sum up, no matter in which perspective, as long as these techniques and techniques are used reasonably, the spatial sense of the picture can be better reflected.

\section{THE EFFECT OF THE USE OF PERSPECTIVE IN THE SCENE ON THE VISUAL EFFECT OF THE AUDIENCE}

Animation requires an integration of various forms of artistic presentation. So just creating a physical sense of space is far from enough, and there is also a need to infuse some plot and clues. This requires the spatial sense of the scene, and it is very important to create a psychological atmosphere for the audience.

Atmosphere is only an abstract sensory factor, a comprehensive visual impression. For animated images, the prominent visual center is that the protagonist and his/her environment depict the animation shots from different angles, thus bringing different impressions to the audience. In animated images, some of the majestic and magnificent spaces are usually composed in a balanced and symmetrical way, which is adopted to present a stable, peaceful atmosphere and a sense of order to the audience. For example, the scene designer of the film "Big Fish \& Begonia" skillfully used curvilinear perspective in the Chinese architectural scene, which not only showed the symmetrical beauty and classical charm of Chinese architecture incisively and vividly, but also created a grand, orderly and dignified psychological feeling for the audience.

Therefore, it can be seen that to influence the psychological changes of the audience, and to add psychological space into the animation production, so as to blend the consciousness with the plot, and the emotions and destiny curves of the characters, is an essential factor for the success of a work For all the methods of expression that can enhance the animation's appeal, it is necessary to consider how much effect they 
have on the psychological space, which is also a wonderful way to make the work more attractive.

Generally, the style scenes are shot from a relatively low angle of view, which brings the psychological feeling of looking at the front horizontally or looking down to the audience. In particular, overlooking scenes can give people a commanding visual feeling, and then connected with some distortion and deformation of perspective effect, can present a sense of reining and soaring. On the contrary, the perspective of looking up is mostly presented from a first-person perspective, often used in snooping or humble roles, where the sense of substitution is strong. The use of large viewing angles, however, is mostly reflected in the commanding plots of the main characters, and is often used in thrillers and action films. In the using process, some only played a role in the narrative of the story, and do nothing to render the atmosphere, so the specific use is up to the director's specific arrangements. An excellent scene designer will balance the relationship between effect and technique and will not use it for the sake of using.

\section{THE RELATIONSHIP BETWEEN PERSPECTIVE AND THE STYLE OF ANIMATION WORKS}

The style of animation production mainly depends on the script, the theme and the director's aesthetic and ideological depth. The style of animation is generally divided into realistic, cartoon and comprehensive experimental types.

The realistic style can be explained according to the literal meaning, that is, people and things in people's real life are taken as the object of creation, and the real world is presented after artistic extraction and refinement. The realistic style is applied more in themes of history and reality, etc. In the realistic style, the use of scene perspective is relatively intuitive and real. The realistic style has always been a form favored by the public, because it can simply and directly show the visual effect.

Cartoon style is more favored by young children. The biggest characteristic is that the picture is relatively simple and easy to understand, the characters are highly recognizable, and it has certain educational significance. Cartoon style scenes are relatively simple, and even part of the scenes are very abstract. Cartoons such as "Tom and Jerry", "SpongeBob SquarePants" and "Peppa Pig", etc. are very popular with children, the scenes of which all show the characteristics of simplicity and abstraction.

The last comprehensive experimental style animation is a relatively niche animation style. The painting style of such works often shows the strong personality of art, and tends to explore and experiment on art rather than for the purpose of profit. So in the market of animation products, such works are rarely enough to see, as they belong to a non-mainstream animation art style. The representative works of this kind include "Song of the Sea " and so on. The creation of experimental style animation will not be restricted by various aspects and there are many materials that can be used. Moreover, the application of scene perspective in the creation of such animation is generally irregular.

The storyline developed around the protagonist is the soul of the animation, and the excellent animation image works are often integrated into the perspective scenes with visual strength. This paper makes exploration and analysis on the perspective of the animation scene and its influence on the overall animation work and how to create a better sense of scene space for the characters from the aspects of theory and practice. Thus, it provides theoretical support for creating more excellent works with visual impact and the development of China's animation industry.

\section{CONCLUSION}

The elaboration of the story is the soul of the whole animation, and the scene is the stage of the characters, which is used to foil the characters and deepen the theme. Summarizing the forms and types of scenes, and explaining their application scope and effects, can play a great role in promoting the development of animation. This paper analyzes the influence of scene perspective on the animation atmosphere and summarizes the role of scene in creating a sense of space and psychological space in the animation, so as to provide more valuable reference for Chinese animation creation.

\section{References}

[1] Zhu Jing, Hu Chuanjiang. Expression of Visual art Language in Animation Scene Design [J]. Art Education Research, 2016 (09): 93. (in Chinese)

[2] Zhang Qing. A brief Analysis of Spatial Design Features of 3D Animation Scenes [J]. Home Drama, 2018 (17): 94-95. (in Chinese)

[3] zhao Jingjing. Research on Application of Multimedia Interactive Technology in Animation Scene Teaching [J]. Art Education Research, 2018 (15): 119. (in Chinese)

[4] Tian Bing. Construction of Perspective Teaching Content Under the Background of Animation Major [J]. Art Education Research, 2018 (08): 100. (in Chinese)

[5] Lu Xiaojie, Ma Jianjun. Information Elements in Animation Shooting Design [J]. Journal of Yancheng Teacher's University (Humanities and Social Sciences Edition), 2017, 37 (03): 106108. (in Chinese)

[6] Zhong Lingqing. Research on the Drawing Method of Fivepoint Perspective in Animation Art Creation [J]. Hunan Packaging, 2016, 31 (03): 30-31 + 57. (in Chinese) 